\title{
Relación entre autoeficacia, apoyo social, adherencia al tratamiento y HbA1C por nivel de percepción de amenaza en pacientes con DM2 Relationship between self-efficacy, social support, treatment adherence and HbA1C by risk perception level in DM2 patients
}

\author{
Anastacia Alhelí Sánchez Arellano ${ }^{1}$, Gabriela Navarro Contreras ${ }^{2}$, Ferrán Padrós Blázquez ${ }^{1}$ y \\ Christian Enrique Cruz Torres ${ }^{2}$
}

Palabras clave: autoeficacia al tratamiento; apoyo social; percepción de amenaza; adherencia al tratamiento; diabetes mellitus; salud; medicina; tratamientos médicos; México Keywords: treatment self-efficacy; social support; risk perception; adherence to treatment; mellitus diabetes; health; medicine; medical treatments; Mexico

Recibido en: 02-04-2020 / Aceptado en: 12-05-2020

\section{Resumen}

Introducción: El principal problema de los pacientes con Diabetes Mellitus Tipo 2 (DM2) es la falta de adherencia a los tratamientos médicos (OMS, 2004). Estos son complejos y exigen realizar modificaciones en el estilo de vida y hábitos de salud. Por esto, el objetivo de esta investigación fue analizar la relación entre autoeficacia, apoyo social para la adherencia al tratamiento y la hemoglobina glucosilada, en función del nivel de percepción de amenaza en pacientes con DM2.

Método: El diseño fue transeccional correlacional con un muestreo no probabilístico por conveniencia, con 166 participantes con DM2 que acuden a atención médica en la Secretaría de Salud y el ISSSTE en Michoacán, México. Se aplicaron instrumentos para evaluar Autoeficacia al Tratamiento en DM2, Apoyo Social en la DM2, las subescalas de severidad y de susceptibilidad percibida de la ECS-DM2/PG y la Escala de adherencia al tratamiento de la DM2.

Resultados: Al dividir la muestra por niveles de percepción de amenaza se puede observar un claro papel mediador de la percepción de susceptibilidad y severidad en el resto de las variables bajo estudio. Manteniendo como variables dependientes la adherencia al tratamiento y la hemoglobina glucosilada, se realizaron análisis de regresión lineal múltiple con el método por pasos para identificar el efecto conjunto de las variables independientes. Los niveles más altos de predicción, tanto para la adherencia al tratamiento como para los niveles de hemoglobina glucosilada se ubican en el grupo con niveles medios de susceptibilidad, donde el $46 \%$ de la varianza de la adherencia al tratamiento se explica con base en la única variable independiente apoyo social percibido. Para los niveles de hemoglobina glucosilada, la predicción de un $33 \%$ de la varianza en el grupo medio

\footnotetext{
${ }^{1}$ Universidad Michoacana de San Nicolás de Hidalgo

${ }^{2}$ Universidad de Guanajuato. E-mail: g.navarro@ugto.mx

(C) Universidad De La Salle Bajío (México)
} 
Relación entre autoeficacia, apoyo social, adherencia al tratamiento y HbA1C por nivel de percepción de amenaza en pacientes con DM2

está dado por los factores higiene, autocuidado y dieta como componentes de la adherencia al tratamiento.

Discusión o Conclusión: Los resultados obtenidos parecen señalar que la relación entre la amenaza percibida y la adherencia no es lineal, de modo que el impacto que tiene la amenaza percibida, cuando el paciente percibe un nivel muy elevado susceptibilidad y severidad parece observarse una tendencia al abandono y al no seguimiento de las indicaciones médicas, al igual que cuando los niveles son muy bajos. También resulta importante tomar en cuenta la percepción de apoyo social, el nivel de apoyo social y la autoeficacia para incrementar la adherencia al tratamiento en los pacientes con DM2.

\begin{abstract}
Introduction: The main problem presented by patients with DM2 is the lack of adherence to medical treatments (WHO, 2004) since it is complex and requires changes in lifestyle and health habits. Objective: analyze the relationship between self-efficacy, social support, and threat perception to treatment adherence.

Method: The design was transectional correlational with a non-probabilistic convenience sampling of 166 participants with DM2 who attended for medical attention at the Ministry of Health and ISSSTE of Michoacán. Instruments were administered to evaluate Self-efficacy to Treatment in DM2, Social Support in DM2, the subscales of severity and perceived susceptibility of the ECSDM2 / PG and the adherence scale to the treatment of DM2.

Results: By dividing the sample by levels of threat perception, a clear mediating role of perception of susceptibility and severity can be observed in the rest of the variables under study. Maintaining treatment adherence and glycosylated hemoglobin as dependent variables, multiple linear regression analyzes were performed using the stepwise method to identify the joint effect of the independent variables. The highest levels of prediction, both for treatment adherence and for glycosylated hemoglobin levels, are in the group with medium levels of susceptibility, where $46 \%$ of the variance of treatment adherence is explained based on the sole independent variable of perceived social support. For glycosylated hemoglobin levels, the prediction of $33 \%$ of the variance in the middle group is given by the factors of hygiene, self-care, and diet as components of treatment adherence.
\end{abstract}

$\mathrm{N}^{\circ}$ 25, Vol. 12 (2), 2020. ISSN 2007 - 0705, pp.: 1 - 22

$$
-2 \text { - }
$$


Discussion or Conclusion: According to the results obtained, it seems that the relationship between the perceived threat and adherence is not linear. It appears to be observed that perceived threat may produce a tendency to abandon by not following medical indications, when the patient perceives a very high level of susceptibility and severity, as well as when the levels are very low. It is also important to consider the perception of social support, the level of social support and selfefficacy to increase adherence to treatment in patients with T2DM.

\section{Introducción}

La complejidad de la adherencia al tratamiento y los múltiples factores que se asocian a ésta dificultan que los pacientes con Diabetes Mellitus tipo 2 (DM2) realicen las recomendaciones médicas (Di Matteo, 2004). La baja adherencia terapéutica tiene como consecuencia directa una alta prevalencia de padecimientos comórbidos que afectan la calidad de vida de los individuos (Boye, Curtis, Lage \& García-Pérez, 2016; Huber \& Reich, 2016). Esto ha motivado la realización de varios estudios (González, Shreck, Psaros \& Safren, 2015) con la finalidad de conocer qué variables se relacionan con un mejor seguimiento de las indicaciones médicas.

Por ejemplo, Al-Khawaldeh, Al-Hassan y Froelicher (2012), reportaron que los sujetos con altos niveles de autoeficacia mostraron más conductas de automanejo en la dieta, ejercicio, automonitoreo de glucosa en sangre y toma de medicamentos. Se encontró que la mayoría de los sujetos que no tienen controlada su diabetes tenían bajos niveles de autoeficacia y conductas no óptimas para su automanejo. Canales y Barra (2014) reportaron que la autoeficacia se relaciona significativamente con la adherencia al tratamiento, ya que como lo refieren otros autores (Essery, Geraghty, Kirb, \& Yardley, 2017), es una variable mediadora para los comportamientos saludables y de adherencia al tratamiento.

La adherencia al tratamiento implica diferentes conductas de salud. Al respecto, cabe mencionar que se han propuesto algunos modelos de predicción que auguran la ocurrencia de conductas relacionadas con la salud (Ortiz, 2013). Uno de estos modelos es el de Creencias en Salud (MCS) el cual se aplicó en un principio a los comportamientos preventivos, pero luego se amplió con éxito para identificar qué aspectos son los que permiten que los pacientes se adhieran al tratamiento médico (Abraham \& Sheeran, 2015). 
En el MCS la percepción de amenaza es un componente del cual depende que se realice una conducta de protección a la salud. Implica dos elementos fundamentales: la percepción de severidad, es decir, qué tan grave es percibida la enfermedad; y la susceptibilidad percibida, que se refiere a la probabilidad que estima el individuo de padecer la enfermedad (Flórez, 2007).

La percepción de amenaza puede ayudar a establecer la relación entre la DM2, las conductas de autocuidado y sus posibles complicaciones. Por lo que evaluarla puede ayudar a explicar cómo los individuos con DM2 perciben tanto su enfermedad, como su tratamiento, y cómo la percepción de amenaza afecta la adherencia (Shreck, Gonzalez, Cohen \& Walker, 2013).

Existen evidencias de que una baja percepción de gravedad de la enfermedad, además de percepción de baja probabilidad de padecerla, pueden determinar los bajos niveles de adherencia observados en pacientes con DM2 (Yeong, 2004). De acuerdo con el MCS (Patino, Sánchez, Eidson \& Delamater, 2005) la percepción de amenaza implica que un individuo pueda reconocer el riesgo que tiene para presentar complicaciones y la gravedad de estas, lo cual motiva a adoptar conductas de salud preventivas.

Otra variable que se ha relacionado con un nivel adecuado de adherencia al tratamiento es el apoyo social (Vinaccia, Contreras, Restrepo, Cadena \& Anaya, 2005; Canales \& Barra, 2014). Como estrategia para mejorar el apoyo social en pacientes con padecimientos crónicos, éstos son entrenados en habilidades para buscar y solicitar apoyo dentro de su familia o comunidad (Steed, Cooke \& Newman, 2003). También se han realizado estudios donde se brinda psicoeducación a los familiares y a los miembros de la comunidad acerca del padecimiento (Nasmith, Cote \& Cox, 2004). Asimismo, se han incorporado a los miembros de la familia o comunidad a los programas de intervención con el objetivo de modificar las conductas de apoyo social que proporcionan, con la finalidad de fomentar un mejor autocuidado en el paciente con diabetes mellitus (Del Castillo, Solano, Iglesias \& Guzmán, 2014).

Mas aún, en la literatura se ha reportado cierta evidencia de la relación entre la autoeficacia, el apoyo social y la percepción de amenaza con la adherencia al tratamiento en pacientes con DM2 (Al-Khawaldeh, et al., 2012; Karimy, Araban, Zareban, Taher \& Abedi ,2016; Canales \& Barra, 2014; Dehghani-Tafti et al., 2015; Pérez \& Bautista, 2008; Vinaccia, Contreras, Restrepo, Cadena y Anaya, 2005). Sin embargo, no se han hallado estudios previos que pongan a prueba el impacto de las variables autoeficacia, apoyo social, percepción de amenaza (susceptibilidad y severidad percibida) en conjunto, en la adherencia terapéutica y la hemoglobina glucosilada. 
Debe considerarse además que nuestro país presenta una de las más altas tasas de DM2 en el mundo, donde el $10.3 \%$, es decir 8.6 millones de adultos, tienen el diagnóstico de DM2 (Instituto Nacional de Salud Pública, 2018), y específicamente en el estado de Michoacán se considera a la DM como la segunda causa de muerte. A su vez, los recursos en salud son muy escasos, y la oferta de tratamiento psicológicos es limitado. Por ello, es muy importante diseñar intervenciones breves

que incidan en aquellas variables que muestren mayor evidencia de relación con la adherencia al tratamiento y la hemoglobina glucosilada.

De modo que conocer el impacto de la autoeficacia, el apoyo social, la susceptibilidad y la severidad percibidas en la adherencia al tratamiento y en la hemoglobina glucosilada, permitirá diseñar estrategias psicológicas efectivas, que se centren en las variables psicológicas más relacionadas con el seguimiento de las indicaciones médicas y en consecuencia un mejor control metabólico que redunde en una disminución de las complicaciones asociadas al padecimiento, tanto a mediano como a largo plazo, aumentando la esperanza y la calidad de vida de los pacientes.

Por estos motivos, el objetivo del presente trabajo fue analizar la relación entre la autoeficacia, el apoyo social, la percepción de amenaza (susceptibilidad y severidad percibida) con la adherencia al tratamiento y con la hemoglobina glucosilada.

\section{Método}

\section{Participantes}

La muestra total fue de 166 participantes mayores de 18 años con diagnóstico confirmado de DM2 por el servicio de Medicina Interna de las unidades de la Secretaría de Salud y del ISSSTE de Michoacán, a las que asisten para recibir atención. El tipo de muestreo fue no probabilístico por conveniencia. El $21.1 \%$ de los participantes fueron hombres y el $78.9 \%$ mujeres, con un rango de edad de 18 a 87 años $(\overline{\mathrm{x}}=59.70$ años, $D E .=12.52$ años $)$.

\section{Instrumentos}

Se administraron los siguientes instrumentos:

1) Autoeficacia al tratamiento en diabetes (Del Castillo, 2010). Mide la autoeficacia que los pacientes perciben para realizar las conductas señaladas en su tratamiento, incluyendo toma de medicamento, seguimiento del plan alimenticio y realización de actividad física. Es una escala tipo Likert con cuatro opciones de respuesta que van de 1 "No me siento capaz" a 4 "Me siento muy 
capaz”. La puntuación máxima de la escala total es de 56 puntos y la mínima es de 14. Los puntajes próximos a 56 hacen referencia que un paciente se siente muy capaz de realizar las conductas señaladas en su tratamiento, las puntuaciones que se encuentran entre 40 a 48 puntos indican que existen niveles moderados de autoeficacia. Los puntajes que se acercan a la puntuación mínima indican que los pacientes no se sienten capaces de seguir el tratamiento indicado por el médico.

El instrumento está conformado por 14 ítems, distribuidos en los siguientes factores: F1. Autoeficacia en el seguimiento del plan alimenticio. Cuenta con un alfa $(\alpha)$ de Cronbach de .78. Los pacientes con puntuaciones altas se sienten capaces de seguir la dieta que sugiere el médico dentro y fuera de su casa, los pacientes con bajas puntuaciones no se sienten capaces de evitar los alimentos que no están en su dieta. F2. Autoeficacia en la realización de actividad física. Tiene un $\alpha$ de Cronbach de .80. Puntuaciones altas en este factor hace referencia que los pacientes se sienten capaces de realizar ejercicio a pesar de que se presenten dificultades para llevarlo a cabo, por ejemplo, cuando el paciente está ocupado. Las puntuaciones bajas en este factor indican que el paciente no se siente capaz de realizar actividad física cuando existen eventualidades en su rutina cotidiana, por ejemplo, sin la compañía de su familia o amigos, o si hace frío o está lloviendo. Por último, el factor tres: F3. Autoeficacia en la toma de medicamentos orales. Cuenta con un alfa de Cronbach de .63. Las puntuaciones altas en este factor refieren que el paciente se siente capaz de recordar tomar sus pastillas todos los días. Las puntuaciones bajas hacen referencia a que el paciente no se siente capaz de seguir el tratamiento médico todos los días. La confiabilidad de la escala total obtenida en población mexicana muestra un $\alpha$ de 0.82 (Del Castillo, 2010).

2) Escala de Apoyo social en la diabetes (Del Castillo, 2010). La escala consta de 27 reactivos que evalúan dos dimensiones: a) la frecuencia; y b) la percepción de apoyo social. La escala total tiene una consistencia interna de Alfa de Cronbach $\alpha=.91$.

La dimensión de frecuencia de apoyo social informa qué tan frecuentemente los pacientes reciben apoyo por parte de sus familiares para seguir su tratamiento, la puntuación máxima es de 68 y la mínima es de 17.

Puntuaciones que se aproximan al 68 hacen referencia a que los familiares brindan apoyo de manera constante a los pacientes con DM2 para realizar las conductas que se establecen en el tratamiento médico, por ejemplo, que los acompañen a sus consultas médicas, que los motiven a realizar ejercicio, que les recuerden tomar su medicamento, etc. En cambio, puntuaciones que se aproximan al 17 indican que los familiares proporcionan poco o nulo apoyo a los pacientes. Esta 
dimensión está constituida por los siguientes factores; Apoyo social emocional $(\alpha=.81)$, apoyo social en la realización de actividad física $(\alpha=.77)$, apoyo social en plan alimenticio $(\alpha=.76)$ y apoyo social en toma de medicamentos $(\alpha=.64)$.

La segunda dimensión denominada Percepción de apoyo social, se refiere a que tan agradable es para los pacientes el apoyo que reciben de los familiares. Tiene una puntuación máxima de 84 y una mínima de 21. Puntuaciones que se aproximan al 84 refieren que para los pacientes es agradable que los familiares los apoyen a realizar las actividades indicadas en su tratamiento, como hacer ejercicio con ellos, los tomen en cuenta para comprar los alimentos que consumirán, los acompañen a sus citas médicas, etc. Puntuaciones que se aproximan al 21 refiere que para los pacientes no es agradable el apoyo que reciben de sus familiares para seguir su tratamiento como es que los regañen cuando no hacen ejercicio, que les impidan decidir qué comer, que les recuerden seguir con su dieta, que los regañen cuando comen algo que no debe comer, que vigilen que comen, etc.

Esta dimensión está conformada por los siguientes factores: Percepción de apoyo social emocional $(\alpha=.93)$, Percepción de apoyo al plan alimenticio $1(\alpha=.76)$, Percepción de regaño $(\alpha$ $=.77)$ y Percepción de apoyo al plan alimenticio $2(\alpha=.78)$.

\section{3) Escala de Creencias en Salud-DM2/Versión paciente Geriátrico (ECS-DM2/PG;}

Domínguez-Guedea, 2006, citado en Madero, 2009). Esta escala tiene como objetivo recolectar información de adultos mayores respecto de sus creencias con relación a la DM2 siguiendo el modelo de creencias de la salud. Para esta investigación se tomaron exclusivamente las subescalas de susceptibilidad y severidad percibida.

La subescala de susceptibilidad percibida tiene una puntuación mínima de 7 y una máxima de 28. Los puntajes que se aproximan al 28 hacen referencia a que los pacientes se sienten susceptibles de padecer las complicaciones generadas por la diabetes a consecuencia de no seguir el tratamiento indicado, en cambio puntuaciones bajas ponen de manifiesto que los pacientes consideran que ellos no van a presentar las complicaciones. Consta de siete ítems con un formato de respuesta tipo Likert que va de 1 "Nada probable, al 4 "Totalmente probable" y cuenta con un de Alfa de Cronbach $\alpha=.84$.

La subescala de severidad percibida tiene una puntuación mínima de 7 y una máxima de 28. Las puntuaciones que se aproximan al 28 hacen referencia a los pacientes consideran que es grave tener complicaciones generadas por la diabetes; los pacientes con puntuaciones bajas 
consideran que las complicaciones no son graves. Está compuesta de siete ítems, las respuestas van de 4 (Muy grave) a 1(Nada grave), con un alfa de Cronbach de .90, los datos de confiabilidad y validez se obtuvieron en población mexicana (Sánchez-Arellano, Navarro y Padrós, 2017).

\section{4) Escala de adherencia al tratamiento de la DM2, versión III (EATDM-III) (Villalobos \&} Araya, 2001). El instrumento está orientado a medir siete factores que indican el nivel de adherencia integral del paciente con Diabetes Mellitus 2. Consta de 55 ítems en formato tipo Likert, que evalúan la frecuencia de ejecución a través de cinco opciones de respuesta que van del cero al cuatro, donde 0 = "Nunca lo hago", hasta el 4 = "Siempre lo hago", mismos que se agrupan en siete factores:

F1. Apoyo Familiar $(\alpha=.83)$, los ítems indican la relación de la familia sobre la condición médica de la persona con Diabetes Mellitus II.

F2. Organización y Apoyo Comunal ( $\alpha=.83$ ), el factor incluye ítems que abordan el tipo de apoyo que la comunidad le brinda a la persona con Diabetes Mellitus II, también hace referencia a la participación de esas personas en las actividades que organiza la comunidad. F3. Ejercicio Físico $(\alpha=.82)$. Incluye ítems que miden la emisión de comportamientos vinculados con actividad física: ejercicio, mantenimiento de horarios para realizarlo y la percepción que se tiene del cuerpo al desarrollar el ejercicio y su relación con el tratamiento médico.

F4. Control Médico $(\alpha=.78)$, está compuesto por ítems que miden el tipo, lugar y las conductas relacionadas con el control médico y preventivo de la Diabetes Mellitus II.

F5. Higiene y Autocuidado $(\alpha=.64)$. Aborda ítems que mezclan conductas vinculadas al mantenimiento de la dieta y conductas de autocuidado específico (por ejemplo: lavarse los pies, utilizar los medicamentos apropiados).

F6. Dieta $(\alpha=.66)$. Incluye los ítems que giran sobre la dieta que deben consumir las personas con Diabetes Mellitus II.

F7. Valoración de la Condición física $(\alpha=.78)$, aborda dos ítems que describen la relación entre ejercicio físico y percepción sobre la condición física. El puntaje máximo de la escala es de 100 el puntaje mínimo es 0 . Puntajes próximos a 100 indican un auto reporte más cercano a pautas de adherencia esperadas, para una vida saludable en personas con DM2. 


\section{Procedimiento}

El protocolo de investigación se sometió a la evaluación del comité de bioética de cada unidad hospitalaria y el comité de ética de la Facultad de Psicología de la UMSNH para su autorización. Los pacientes que cumplieron con los criterios de inclusión y que estuvieron de acuerdo en participar en la investigación dieron su consentimiento por escrito. Se les aplicó la batería de pruebas de forma individual en una sola sesión de aproximadamente 45 minutos.

Después de tener los datos de los participantes, se le solicitó a la unidad médica el dato más reciente de hemoglobina glucosilada (HbA1c) de cada participante. La HbA1c indica por medio de un porcentaje el promedio de la concentración de glucemia en la sangre de los últimos tres meses antes de la muestra (Campuzano-Maya \& Latorre-Sierra, 2010). La meta de control glucémico, de acuerdo con la Asociación Latinoamericana de Diabetes (2013), es un rango no mayor a 7 \%. Los participantes dieron su autorización para proporcionar esta información en el consentimiento informado.

\section{$\underline{\text { Resultados }}$}

El tiempo promedio de diagnóstico con la DM2 de los participantes fue de 11.57 años (D.E. = 7.58 años) y el promedio en Hemoglobina Glucosilada (HbAc1) que obtuvieron fue de 8.3 (D.E. = 2.2), promedio por arriba del porcentaje recomendado para un adecuado control glucémico.

Como puede observarse en la Tabla 1, la adherencia al tratamiento tuvo una correlación estadísticamente significativa y positiva con la percepción de apoyo social, el nivel de apoyo social y la autoeficacia al tratamiento. Pero no se encontraron correlaciones significativas entre la adherencia al tratamiento y la severidad percibida, ni con la susceptibilidad percibida. 
Tabla 1. Correlaciones de Spearman de las variables en estudio

Table 1. Spearman correlations of variables under study.

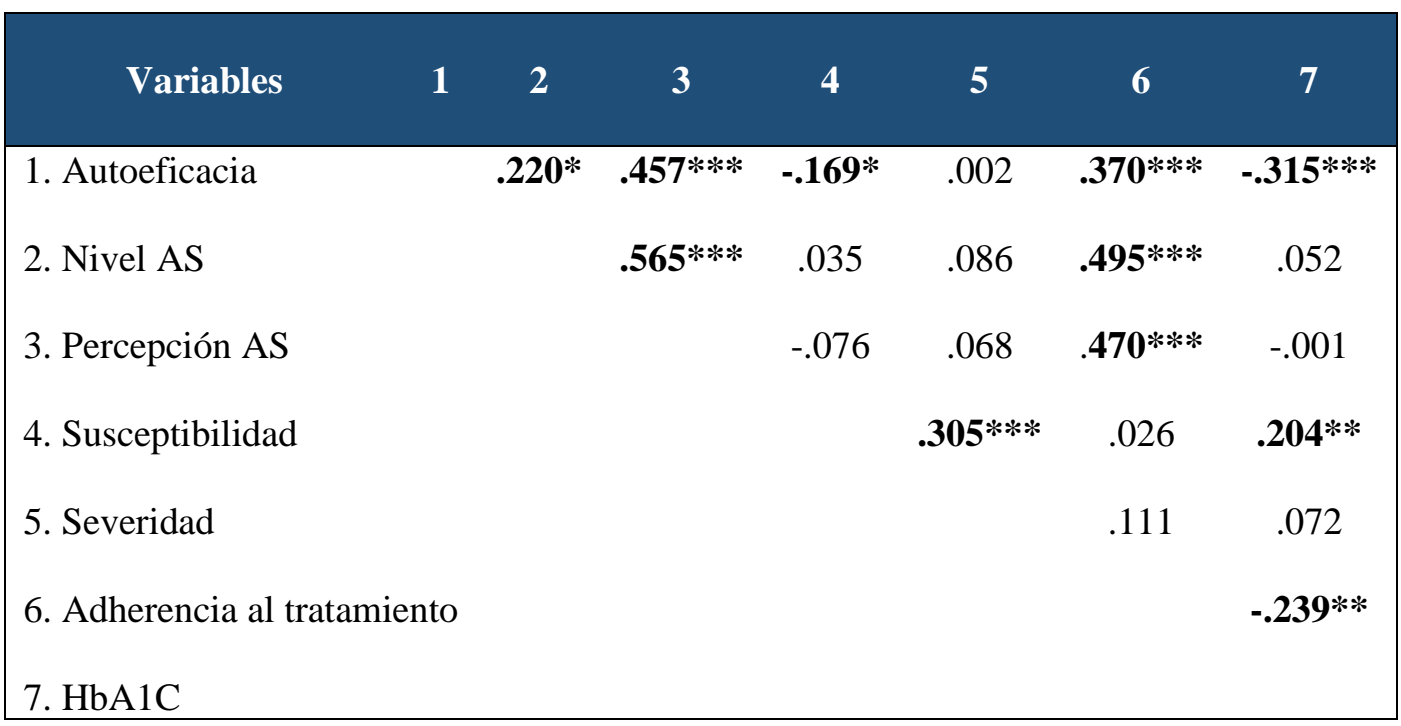

Nota: Nivel AS = Nivel de Apoyo Social; Percepción AS = Percepción de Apoyo Social; HbA1c = Hemoglobina glucosilada.

$* * * \mathrm{p}<0.001 ; * * \mathrm{p}<0.01 ; * \mathrm{p}<0.05$

Note: Level AS = Level of Social Support; Perception AS = Perception of Social Support; HbA1c = Glycated hemoglobin.

$* * * \mathrm{p}<0.001 ; * * \mathrm{p}<0.01 ; * \mathrm{p}<0.05$

Considerando la hipótesis de la susceptibilidad y la severidad percibidas como variables mediadoras entre las variables independientes (i.e. autoeficacia, nivel de apoyo social, percepción de apoyo social) y las dependientes (i.e. adherencia al tratamiento y hemoglobina glucosilada), se dividió a la muestra de acuerdo con sus puntajes, en grupos bajos (susceptibilidad<14; severidad <20.66), medios (susceptibilidad >14 y <18; severidad >20.66 y <26) y altos (susceptibilidad>18; severidad >26).

Se realizaron correlaciones de Spearman por nivel de susceptibilidad percibida y por nivel de severidad percibida. Como se puede observar en las Tablas 2 y 3, las correlaciones de las variables bajo estudio se modifican de acuerdo con el nivel de percepción de susceptibilidad y severidad que presenta el participante, siendo en los grupos medios y altos donde se observan mayores correlaciones y en mayor número.

En la Tabla 2 se puede observar que la adherencia al tratamiento tuvo una correlación estadísticamente significativa y positiva en el grupo bajo con la percepción de apoyo social y la autoeficacia al tratamiento. El grupo medio correlacionó en ese mismo sentido con el nivel de apoyo social y percepción de apoyo social. Mientras que en el grupo de alta susceptibilidad 
percibida hubo correlaciones estadísticamente significativas y positivas con todas las variables, excepto con la HbA1C, que como se esperaba correlacionó de manera inversa. La Autoeficacia obtuvo correlaciones estadísticamente significativas y negativas en los grupos bajo y alto con HbA1C y positivas con la adherencia al tratamiento, en estos mismos grupos, pero no se encontró correlación en el grupo de nivel medio de susceptibilidad percibida.

Tabla 2. Correlaciones de Spearman de las variables en estudio por nivel de susceptibilidad percibida

Table 2. Spearman correlations of variables under study by level of perceived susceptibility

\begin{tabular}{|c|c|c|c|c|c|}
\hline NSP & Variables & 2 & 3 & 4 & 5 \\
\hline \multirow{5}{*}{ 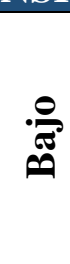 } & 1. Autoeficacia & -.094 & .204 & $.479^{* * * *}$ & $-.403^{* * *}$ \\
\hline & 2. Nivel AS & & $.470^{* * * * *}-100$ & .219 & 261 \\
\hline & 3. Percepción AS & & & $.312^{*}$ & .137 \\
\hline & 4. Adherencia al tratamiento & & & & -.210 \\
\hline & 5. $\mathrm{HbA1C}$ & & & & \\
\hline \multirow{5}{*}{ 冚 } & 1. Autoeficacia & $.454^{* * * *}$ & $.540^{* * * *}$ & .254 & -.181 \\
\hline & 2. Nivel AS & & $.732^{* * * *}$ & $.686^{* * * *}$ & -.001 \\
\hline & 3. Percepción AS & & & $.634^{* * * *}$ & .009 \\
\hline & 4. Adherencia al tratamiento & & & & -.248 \\
\hline & 5. $\mathrm{HbA1C}$ & & & & \\
\hline \multirow{5}{*}{$\stackrel{e}{\sharp}$} & 1. Autoeficacia & $.290^{*}$ & $.364^{* *}$ & $.416^{* *}$ & $-.384^{* *}$ \\
\hline & 2. Nivel AS & & $.433^{* *}$ & $.502^{* * * *}$ & .009 \\
\hline & 3. Percepción AS & & & $.431^{* *}$ & -.032 \\
\hline & 4. Adherencia al tratamiento & & & & -.228 \\
\hline & 5. $\mathrm{HbA} 1 \mathrm{C}$ & & & & \\
\hline
\end{tabular}

Nota: Nivel de susceptibilidad percibida = NSP; Nivel AS = Nivel de Apoyo Social; Percepción AS = Percepción de Apoyo Social; HbA1c = Hemoglobina glucosilada. $* * * \mathrm{p}<0.001 ; * * \mathrm{p}<0.01 ; * \mathrm{p}<0.05$

Note: Level of perceived susceptibility $=$ NSP; Level AS $=$ Level of Social Support; Perception AS = Perception of Social Support; HbA1c $=$ Glycated hemoglobin . $* * * \mathrm{p}<0.001 ; * * \mathrm{p}<0.01 ; * \mathrm{p}<0.05$

Respecto a la severidad percibida encontramos, como se observa en la Tabla 3, correlaciones estadísticamente significativas y negativas entre autoeficacia y $\mathrm{HbA1C}$ en el grupo bajo y medio. Por otro lado, se observan correlaciones positivas entre autoeficacia y la percepción de apoyo social en los tres niveles. Con la percepción de apoyo social en los niveles medio y alto, y con el nivel de apoyo social solo en el grupo alto. La adherencia al tratamiento obtuvo correlaciones 
estadísticamente significativas y positivas con todas las variables y en todos los niveles de severidad, excepto con la autoeficacia en el nivel bajo de severidad.

Tabla 3. Correlaciones de Spearman de las variables en estudio por nivel de Severidad percibida.

Table 3. Spearman correlations of variables under study by level of perceived severity.

\begin{tabular}{|c|c|c|c|c|c|}
\hline NSeP & Variables & 2 & 3 & 4 & 5 \\
\hline \multirow{5}{*}{ •ִ } & 1. Autoeficacia & .019 & $.463 * *$ & .216 & $-.354 * *$ \\
\hline & 2. Nivel AS & & $.590^{* * * *}$ & $.416 *$ & .033 \\
\hline & 3. Percepción AS & & & $.438 * *$ & -.112 \\
\hline & 4. Adherencia al tratamiento & & & & -.193 \\
\hline & 5. $\mathrm{HbA} 1 \mathrm{C}$ & & & & \\
\hline \multirow{4}{*}{ 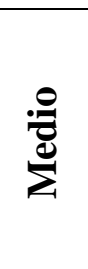 } & 1. Autoeficacia & .226 & $.340^{* *}$ & $.377 * *$ & $-.291 *$ \\
\hline & 2. Nivel AS & & $.489^{* * * *}$ & $.486^{* * * *}$ & .018 \\
\hline & 3. Percepción AS & & & $.542 * * *$ & .073 \\
\hline & $\begin{array}{l}\text { 4. Adherencia al tratamiento } \\
\text { 5. HbA1C }\end{array}$ & & & & -.209 \\
\hline \multirow{4}{*}{$\stackrel{8}{\gtrless}$} & 1. Autoeficacia & $.342^{*}$ & $.502 * * *$ & $.470^{* * * *}$ & -.242 \\
\hline & 2. Nivel AS & & $.618^{* * *}$ & $.572 * * *$ & .194 \\
\hline & 3. Percepción AS & & & $.378^{* * *}$ & .002 \\
\hline & $\begin{array}{l}\text { 4. Adherencia al tratamiento } \\
\text { 5. HbA1C }\end{array}$ & & & & -.240 \\
\hline
\end{tabular}

Nota: Nivel de severidad Percibida = NSeP; Nivel AS = Nivel de Apoyo Social; Percepción AS = Percepción de Apoyo Social; HbA1c = Hemoglobina glucosilada.

$* * * \mathrm{p}<0.001 ; * * \mathrm{p}<0.01 ; * \mathrm{p}<0.05$

Note: Perceived severity level $=$ NSeP; Level AS $=$ Level of Social Support; Perception AS = Perception of Social Support; HbA1c = Glycated hemoglobin. $* * * \mathrm{p}<0.001 ; * * \mathrm{p}<0.01 ; * \mathrm{p}<0.05$

Manteniendo como variables dependientes la adherencia al tratamiento y la hemoglobina glucosilada y siguiendo las correlaciones observadas en la Tabla 1, se realizaron análisis de regresión lineal múltiple con el método por pasos para identificar el efecto conjunto de las variables independientes. Para cada análisis se seleccionó el modelo de regresión con los niveles más altos de varianza explicada considerando un incremento significativo de $\mathrm{r}^{2}$.

Como se observa en la Tabla 4, la percepción de apoyo social, el nivel de apoyo social y la autoeficacia explican en conjunto un $32 \%$ de la varianza de la adherencia al tratamiento. Con niveles de tolerancia superiores a .5 y de factor de inflación de varianza inferiores a 2 se asume la ausencia de problemas de colinealidad entre las variables independientes del modelo. Los niveles de Hemoglobina glucosilada son explicados solo en un $13 \%$ de su variación por la autoeficacia y 
la susceptibilidad, siendo descartada del modelo la adherencia al tratamiento por no incrementar significativamente la varianza explicada del modelo $(t=-1.71, p=.08)$. En este modelo llama la atención que el efecto predictor de la susceptibilidad es positivo, implicando que mayor susceptibilidad percibida deriva en niveles más altos de hemoglobina glucosilada.

Al descartarse la variable de adherencia al tratamiento por su escasa capacidad predictiva se exploró la posibilidad de que solo algunos de los factores tuvieran capacidad predictiva sobre la Hemoglobina glucosilada, efecto que se estaría perdiendo al analizar la varianza de todos sus componentes en un indicador global. Para analizar esa posibilidad, se realizó un nuevo análisis de regresión para predecir los niveles de Hemoglobina glucosilada. En este análisis se usaron como variables independientes nuevamente los factores de autoeficacia y susceptibilidad, además de los componentes de adherencia al tratamiento que son: apoyo familiar, organización y apoyo comunal, ejercicio físico, control médico, higiene y autocuidado, dieta, y valoración de la condición física. Como se observa en la última fila de la Tabla 4, se alcanza una mejor explicación de la varianza explicada en este modelo (16\%) derivada de la autoeficacia y el componente higiene y autocuidado de la adherencia al tratamiento. En este nuevo modelo se descarta como predictora la variable susceptibilidad por no contribuir a incrementar significativamente la varianza explicada del modelo, así como todos los demás componentes de la adherencia al tratamiento (todos los valores de $\mathrm{t}<1.78$ con valores de $\mathrm{p}>.07)$.

Tabla 4. Regresiones lineales múltiples para predecir la adherencia al tratamiento y los niveles de hemoglobina glucosilada.

Table 4. Multiple linear regressions to predict adherence to treatment and glycated hemoglobin levels.

\begin{tabular}{|c|c|c|c|c|c|c|c|c|}
\hline $\begin{array}{c}\text { Variable } \\
\text { dependiente }\end{array}$ & $r^{2}$ & $\Delta \mathbf{r}^{2}$ & $\begin{array}{l}\text { Variables } \\
\text { Predictoras }\end{array}$ & $\boldsymbol{\beta}$ & $\mathbf{t}$ & $\mathbf{P}$ & Tolerancia & FIV \\
\hline \multirow{3}{*}{$\begin{array}{l}\text { Adherencia al } \\
\text { tratamiento }\end{array}$} & \multirow{3}{*}{0.32} & \multirow{3}{*}{$\begin{array}{c}.036(\Delta \mathrm{F}=6.78 \\
\mathrm{gl}=1,130 ; \mathrm{p}= \\
.010)\end{array}$} & Percepción AS & 0.22 & 2.18 & 0.03 & 0.51 & 1.96 \\
\hline & & & & 0.28 & 2.89 & 0.00 & 0.57 & 1.7 \\
\hline & & & Auto & 0.21 & 2.60 & 0.01 & 0.85 & 1.1 \\
\hline \multirow{2}{*}{$\mathrm{HbAlc}$} & \multirow{2}{*}{0.13} & $\begin{array}{c}0.025(\Delta \mathrm{F}= \\
4.05 ; \mathrm{gl}=1,140 ;\end{array}$ & Aut & 0.29 & 3.68 & $<.001$ & 0.97 & \\
\hline & & & Susce & 0.16 & 2.01 & 0.05 & 0.97 & 1.0 \\
\hline \multirow{2}{*}{ HbA1c } & \multirow{2}{*}{0.16} & $0.068(\Delta \mathrm{F}=$ & $\mathrm{Au}$ & 0.27 & 3.35 & 0.00 & 0.97 & 1. \\
\hline & & $1,133 ; p=.001)$ & $\begin{array}{c}\text { Adherencia (Higiene } \\
\text { y autocuidado) }\end{array}$ & $\begin{array}{c}- \\
0.27\end{array}$ & $\begin{array}{c}- \\
3.30\end{array}$ & 0.00 & 0.97 & $1 .($ \\
\hline
\end{tabular}

Nota: Nivel AS = Nivel de Apoyo Social; Percepción AS = Percepción de Apoyo Social; HbA1c = Hemoglobina glucosilada; FIV = Factor de inflación de varianza.

Note: Level AS = Level of Social Support; Perception AS = Perception of Social Support; HbA1c = Glycated hemoglobin; FIV = Variance Inflation Factor. 
Posteriormente se realizaron los mismos análisis de regresión para cada grupo por separado para cada nivel (bajo, medio y alto) de severidad y susceptibilidad percibida. Considerando que niveles muy bajos de susceptibilidad y severidad pueden generar baja motivación para el autocuidado, y que niveles muy altos pueden llevar a la negación del problema. Se esperaba que los niveles más altos de predicción de la adherencia al tratamiento y la hemoglobina glucosilada se encontraran en los grupos intermedios.

Como se observa en la Tabla 5, los niveles más altos de predicción, tanto para la adherencia al tratamiento como para los niveles de hemoglobina glucosilada se ubican en el grupo con niveles medios de susceptibilidad, donde el $46 \%$ de la varianza de la adherencia al tratamiento se explica con base en la única variable independiente del apoyo social percibido.

Para los niveles de hemoglobina glucosilada, la predicción de un $33 \%$ de la varianza en el grupo medio está dado por los factores higiene, autocuidado y dieta, como componentes de la adherencia al tratamiento.

Tabla 5. Regresiones lineales múltiples para predecir la adherencia al tratamiento y los niveles de hemoglobina glucosilada en pacientes con niveles bajos, medios y altos de susceptibilidad.

Table 5. Multiple linear regressions to predict adherence to treatment and glycated hemoglobin levels in patients with low, medium, and high susceptibility levels.

\begin{tabular}{|c|c|c|c|c|c|c|c|c|}
\hline $\begin{array}{c}\text { Nivel de } \\
\text { susceptibilidad }\end{array}$ & $\mathbf{r} 2$ & $\Delta \mathbf{r} 2$ & $\begin{array}{l}\text { Variables } \\
\text { Predictoras }\end{array}$ & B & $\mathbf{T}$ & $\mathbf{p}$ & Tolerancia & FIV \\
\hline \multicolumn{9}{|c|}{ Adherencia al tratamiento (dependiente) } \\
\hline \multirow{2}{*}{ Bajo } & \multirow{2}{*}{0.31} & \multirow{2}{*}{$\begin{array}{l}0.087(\Delta \mathrm{F}=5.68 ; \mathrm{gl} \\
\quad=1,45 ; \mathrm{p}=.021)\end{array}$} & \multirow{2}{*}{$\begin{array}{c}\text { Percepción AS } \\
\text { Autoeficacia }\end{array}$} & 0.44 & \multirow{2}{*}{$\begin{array}{l}3.51 \\
2.38\end{array}$} & 0.001 & \multirow{2}{*}{$\begin{array}{l}0.98 \\
0.98\end{array}$} & \multirow{2}{*}{$\begin{array}{l}1.01 \\
1.01\end{array}$} \\
\hline & & & & 0.29 & & 0.021 & & \\
\hline Medio & 0.46 & $\begin{array}{c}0.46(\Delta \mathrm{F}=31.59 ; \mathrm{gl} \\
\quad=1,37 ; \mathrm{p}<.001)\end{array}$ & Nivel AS & 0.67 & 5.62 & 0.001 & 1.00 & 1.00 \\
\hline \multirow{2}{*}{ Alto } & \multirow{2}{*}{0.37} & \multirow{2}{*}{$\begin{array}{l}0.097(\Delta \mathrm{F}=6.49 ; \mathrm{gl} \\
\quad=1,42 ; \mathrm{p}=.015)\end{array}$} & \multirow{2}{*}{$\begin{array}{c}\text { Autoeficacia } \\
\text { Nivel AS }\end{array}$} & 0.32 & \multirow{2}{*}{$\begin{array}{l}2.54 \\
3.53\end{array}$} & 0.010 & \multirow{2}{*}{$\begin{array}{l}0.93 \\
0.93\end{array}$} & \multirow{2}{*}{$\begin{array}{l}1.07 \\
1.07\end{array}$} \\
\hline & & & & 0.44 & & 0.001 & & \\
\hline \multicolumn{9}{|c|}{ Hemoglobina glucosilada (dependiente) } \\
\hline \multirow[b]{2}{*}{ Bajo } & \multirow[b]{2}{*}{0.29} & \multirow{2}{*}{$\begin{array}{c}0.10(\Delta \mathrm{F}=6.34 ; \mathrm{gl}= \\
1,44 ; \mathrm{p}=.015)\end{array}$} & Autoeficacia & -0.38 & \multirow{2}{*}{$\begin{array}{l}-2.94 \\
-2.52\end{array}$} & 0.005 & 0.96 & 1.03 \\
\hline & & & $\begin{array}{l}\text { Adherencia (Control } \\
\text { médico) }\end{array}$ & -0.32 & & 0.015 & 0.96 & 1.03 \\
\hline \multirow{2}{*}{ Medio } & \multirow{2}{*}{0.33} & \multirow{2}{*}{$\begin{array}{c}0.92(\Delta \mathrm{F}=5.67 ; \mathrm{gl}= \\
1,43 ; \mathrm{p}<.022)\end{array}$} & $\begin{array}{c}\text { Adherencia (Higiene } \\
\text { y autocuidado) }\end{array}$ & -0.55 & -4.22 & $<.001$ & 0.96 & 1.03 \\
\hline & & & Adherencia (Dieta) & -0.31 & 2.38 & 0.022 & 0.96 & 1.03 \\
\hline Alto & 0.12 & $\begin{array}{l}0.097(\Delta \mathrm{F}=6.49 ; \mathrm{gl} \\
\quad=1,42 ; \mathrm{p}=.015)\end{array}$ & Autoeficacia & -0.35 & -2.49 & 0.017 & 1.00 & 1.00 \\
\hline
\end{tabular}

Nota: Nivel AS = Nivel de Apoyo Social; Percepción AS = Percepción de Apoyo Social; FIV = Factor de inflación de varianza.

Note: Level AS = Level of Social Support; Perception AS = Perception of Social Support; FIV = Variance Inflation Factor. 
La Tabla 6 muestra también, una mayor explicación de la varianza de los niveles de hemoglobina glucosilada para el grupo medio en severidad. Ahí podemos observar que el $27 \%$ de la varianza está explicado por los factores Control médico e Higiene y autocuidado de adherencia al tratamiento.

Sin embargo, en la predicción de adherencia al tratamiento, los niveles más altos de explicación de varianza se observan en el grupo alto en severidad, con un $44 \%$ de la varianza explicada por la autoeficacia y la percepción de apoyo social.

Tabla 6. Regresiones lineales múltiples para predecir la adherencia al tratamiento y los niveles de hemoglobina glucosilada en pacientes con niveles bajos, medios y altos de severidad percibida.

Table 6. Multiple linear regressions to predict adherence to treatment and glycated hemoglobin levels in patients with low, medium, and high levels of perceived severity.

\begin{tabular}{|c|c|c|c|c|c|c|c|c|}
\hline $\begin{array}{l}\text { Nivel de } \\
\text { severidad }\end{array}$ & $\mathbf{r} 2$ & $\Delta \mathbf{r}$ & Variables predictoras & B & $\mathbf{t}$ & $\mathbf{p}$ & Tolerancia & FIV \\
\hline \multicolumn{9}{|c|}{ Adherencia al tratamiento (dependiente) } \\
\hline Bajo & 0.22 & $\begin{array}{l}0.22(\Delta \mathrm{F}=10.04 ; \mathrm{gl} \\
\quad=1,34 ; \mathrm{p}=.003)\end{array}$ & Percepción AS & 0.47 & 3.17 & 0.003 & 1.00 & 1.00 \\
\hline \multirow{2}{*}{ Medio } & \multirow{2}{*}{0.35} & \multirow{2}{*}{$\begin{array}{l}0.76(\Delta \mathrm{F}=5.034 ; \mathrm{gl} \\
\quad=1,43 ; \mathrm{p}=.03)\end{array}$} & Percepción AS & 0.48 & 3.89 & $<0.001$ & 0.97 & 1.02 \\
\hline & & & Autoeficacia & 0.27 & 2.24 & 0.030 & 0.97 & 1.02 \\
\hline \multirow[b]{2}{*}{ Alto } & \multirow[b]{2}{*}{0.44} & \multirow{2}{*}{$\begin{array}{l}0.083(\Delta \mathrm{F}=6.47 ; \mathrm{gl} \\
\quad=1,43 ; \mathrm{p}=.015)\end{array}$} & Autoeficacia & 0.31 & 2.54 & 0.015 & 0.86 & 1.15 \\
\hline & & & Percepción AS & 0.49 & 4.04 & $<0.001$ & 0.86 & 1.15 \\
\hline \multicolumn{9}{|c|}{ Hemoglobina glucosilada } \\
\hline \multirow{2}{*}{ Bajo } & \multirow{2}{*}{0.26} & \multirow{2}{*}{$\begin{array}{l}0.92(\Delta \mathrm{F}=4.64 ; \mathrm{gl} \\
\quad=1,37 ; \mathrm{p}=.038)\end{array}$} & Adherencia (Dieta) & -0.47 & 3.28 & 0.002 & 0.96 & 1.03 \\
\hline & & & $\begin{array}{l}\text { Adherencia (Control } \\
\text { médico) }\end{array}$ & -0.30 & -2.15 & 0.038 & 0.96 & 1.03 \\
\hline \multirow{2}{*}{ Medio } & \multirow{2}{*}{0.27} & \multirow{2}{*}{$\begin{array}{l}0.06(\Delta \mathrm{F}=4.41 ; \mathrm{gl} \\
\quad=1,47 ; \mathrm{p}=.04)\end{array}$} & $\begin{array}{l}\text { Adherencia (Control } \\
\text { médico) }\end{array}$ & -0.29 & -2.10 & 0.040 & 0.77 & 1.28 \\
\hline & & & $\begin{array}{c}\text { Adherencia (Higiene y } \\
\text { autocuidado) }\end{array}$ & -0.31 & -2.23 & 0.030 & 0.77 & 1.28 \\
\hline \multirow[b]{2}{*}{ Alto } & \multirow[b]{2}{*}{0.24} & \multirow[b]{2}{*}{$\begin{array}{l}0.10(\Delta \mathrm{F}=6.35 ; \mathrm{gl} \\
\quad=1,46 ; \mathrm{p}=.015)\end{array}$} & $\begin{array}{c}\text { Adherencia (Higiene y } \\
\text { autocuidado) }\end{array}$ & -0.31 & -2.52 & 0.015 & 0.99 & 1.01 \\
\hline & & & $\begin{array}{l}\text { Adherencia } \\
\text { (Valoración de la } \\
\text { condición física) }\end{array}$ & -0.39 & -3.11 & 0.003 & 0.99 & 1.01 \\
\hline
\end{tabular}

Nota: Percepción AS = Percepción de Apoyo Social; FIV = Factor de inflación de varianza. Note: Perception AS = Perception of Social Support; FIV = Variance Inflation Factor. 
Relación entre autoeficacia, apoyo social, adherencia al tratamiento y HbA1C por nivel de percepción de amenaza en pacientes con DM2

\section{Discusión}

El concepto de adherencia al tratamiento en la DM2 sigue siendo difuso, lo que dificulta la atención de los pacientes. Dado que se relaciona con múltiples variables psicológicas representa un reto para los psicólogos al diseñar e implementar intervenciones para que los pacientes puedan seguir las recomendaciones médicas. Esto se debe a que la adherencia al tratamiento en la DM2 implica que el paciente realice de manera eficiente múltiples conductas al mismo tiempo, como son el seguimiento de la dieta, monitoreo de glucosa, toma de distintos medicamentos, ejercitarse físicamente, acudir a consultas médicas, revisión de pies y ojos. Cada una de esas conductas tiene su grado de dificultad y a su vez se relacionan con el deterioro que puedan presentar los pacientes por el padecimiento. Aunado a lo anterior, las personas con diabetes deben tomar decisiones de manera cotidiana y rápida sobre su tratamiento, para lo cual un adecuado nivel de autoeficacia y apoyo social pueden ser de gran ayuda.

Los resultados mostraron que la adherencia al tratamiento tuvo una correlación positiva y estadísticamente significativa con la autoeficacia al tratamiento, como se reporta en otros estudios, (González-Cantero, González, Vázquez \& Galán, 2015; Martos-Méndez, 2015; Bonnie \& Doris, 2015; Li Cheng et al., 2016, Devarajooh \& Chinna, 2017).

Los análisis de regresión lineal aquí presentados muestran que la percepción de apoyo social, el nivel de apoyo social y la autoeficacia se tienen que tomar en cuenta para incrementar la adherencia al tratamiento en los pacientes con DM2, ya que explican el $32 \%$ de la varianza de la adherencia al tratamiento. Lo anterior, concuerda con estudios previos (Tang, Brown, Funnell \& Anderson, 2008; Nicklett \& Liang, 2010; Strom \& Egede, 2012), donde el apoyo social positivo, es decir aquel que perciben los pacientes que es benéfico para que puedan seguir las recomendaciones médicas, favorece las conductas relacionadas con el cuidado de la salud en pacientes con DM2.

Así mismo, Martos-Méndez (2015) y King et al. (2010), encontraron que tanto la autoeficacia como la adherencia al tratamiento correlacionan de manera inversa con la Hemoglobina glucosilada, mostrando que a menores puntajes de autoeficacia y menor adherencia al tratamiento peor control glucémico.

Encontramos como resultado del primer modelo de regresión, que el efecto predictor de la susceptibilidad es positivo, implicando que mayor susceptibilidad percibida deriva en niveles más altos de hemoglobina glucosilada. Con respecto a lo anterior, estudios previos han señalado que la 
relación entre percepción de amenaza y el autocuidado en la diabetes es ambigua, (Shreck et al., 2013). De acuerdo con Mills et al. (2008), algunas veces se relaciona positivamente y otras negativamente con las conductas de riesgo y las conductas de autocuidado. Los resultados no consistentes pueden deberse a que en la literatura el concepto de percepción de amenaza se evalúa en múltiples dimensiones y con instrumentos diversos, generando así diferentes resultados.

Por ejemplo, en el estudio de Yeong (2004) se identificó qué existe una relación entre la susceptibilidad y la severidad percibida con la realización de conductas preventivas para las complicaciones relacionadas con la DM2. En ese estudio los pacientes con niveles bajos de percepción de amenaza, que consideraban que la enfermedad no era grave y con poca percepción de susceptibilidad, tenían bajos niveles en las conductas preventivas para las complicaciones. En ese mismo sentido, Pourghaznein et al. (2013) encontraron una correlación positiva de la percepción de severidad y la susceptibilidad percibida con la adherencia al tratamiento. Sin embargo, Shreck et al. (2013), reportaron en su investigación con adultos con DM2 que participaban en una intervención conductual, que no hubo relación entre la percepción de riesgo y control glucémico.

Al parecer, la relación entre la amenaza percibida y la adherencia no es lineal. Al dividir la muestra del presente estudio en grupos altos, medios y bajos en susceptibilidad y severidad percibida pudimos poner en evidencia su papel como variables mediadoras. Encontramos que los niveles más altos de predicción tanto para la adherencia al tratamiento como para los niveles de hemoglobina glucosilada se ubican en el grupo con niveles medios y altos de susceptibilidad.

De este modo, el impacto que tiene la amenaza percibida cuando el paciente percibe un nivel muy elevado sobre todo de severidad parece relacionarse con una tendencia al no seguimiento de las indicaciones médicas e incluso al abandono. Lo anterior puede ocasionar que se presente un menor control metabólico como lo muestran nuestros resultados, al igual que cuando los niveles de la amenaza percibida son muy bajos. Sin embargo, en niveles medios y altos sí se manifiesta una relación positiva, como se mostró en el presente estudio, lo cual concuerda con lo reportado por Pourghaznein et al. (2013).

Llama la atención que el nivel medio de susceptibilidad percibida no correlacione con la HbA1C, mientras tanto en el grupo bajo como en el alto sí, por lo que se sugiere para futuros estudios continuar con el estudio de estas variables. Sería deseable en futuras investigaciones tener 
Relación entre autoeficacia, apoyo social, adherencia al tratamiento y HbA1C por nivel de percepción de amenaza en pacientes con DM2

muestras más amplias de participantes con DM2, donde se pudieran generar grupos diferenciando aquellos con muy alto nivel de percepción de amenaza, muy bajo y niveles moderados.

\section{$\underline{\text { Agradecimientos }}$}

Esta investigación se realizó con apoyo de la beca CONACyT para estudios de posgrado.

\section{$\underline{\text { Referencias }}$}

Al-Khawaldeh, O.A., Al-Hassan, M.A., \& Froelicher, E.S. (2012). Self-efficacy, self-management, and glycemic control in adults with type diabetes mellitus. Journal Diabetes Complications, 26(1), 10-16. DOI: $\underline{10.1016 / j \text { j.jdiacomp.2011.11.002 }}$

Abraham, C., \& Sheeran (2015). The Health Belief Model. (30-69). In M. Conner \& P. Norman (Eds.). Predicting and Changing Health Behavior. Nueva York, USA: McGraw-Hill.

Abubakari, A.R., Cousins, R., Thomas, C., Sharma, D., \& Naderali, E.K. (2016). Sociodemographic and clinical predictors of self-management among people with poorly controlled Type 1 and Type 2 Diabetes: the role of Illness perceptions and self-efficacy. Journal of Diabetes Research, 1-12. DOI: https://doi.org/10.1155/2016/6708164

Asociación Latinoamericana de Diabetes. (2013). Guías ALAD sobre el diagnóstico, control y tratamiento de la Diabetes Mellitus Tipo 2 con Medicina Basada en Evidencia. Recuperado de https://issuu.com/alad-diabetes/docs/guias_alad_2013

Bonnie, A. F, \& Doris, F.G. (2015). The effect of Self- Efficacy on treatment. Journal of Addictions Nursing, 2, 62-70. DOI: 10.1097/JAN.0000000000000073

Boye, K. S., Curtis, S. E., Lage, M. J., \& Garcia-Perez, L. E. (2016). Associations between adherence and outcomes among older, type 2 diabetes patients: evidence from a Medicare Supplemental database. Patient preference and adherence, 10, 1573-1581. DOI: 10.2147/PPA.S107543

Canales, S., \& Barra, E. (2014). Autoeficacia, apoyo social y adherencia al tratamiento en adultos con diabetes mellitus tipo II. Psicología y Salud, 24(2), 167-173. DOI: https://doi.org/10.25009/pys.v24i2.922

Campuzano-Maya, G., \& Latorre-Sierra, G. (2010). La HbA1c en el diagnóstico y en el manejo de la diabetes. Medicina \& Laboratorio, 16, 211-214. 
Dehghani-Tafti, A., Mahmoodabad, S. S. M., Morowatisharifabad, M. A., Ardakani, M. A., Rezaeipan-dari, H., \& Lotfi, M. H. (2015). Determinants of Self-Care in Diabetic Patients Based on Health Belief Model. Global journal of health science, 7(5), 33- 42. DOI: 10.5539/gjhs.v7n5p33

Del Castillo, A. (2010). Apoyo social, síntomas depresivos, autoeficacia y bienestar psicológico en pacientes diabéticos tipo 2. (Tesis Doctoral). Recuperado de http://132.248.9.195/ptb2010/noviembre/0664217/Index.html

Del Castillo, A., Solano, G., Iglesias, S., \& Guzmán, R. (2014). Apoyo familiar en el manejo de la diabetes tipo 2. (108-130). En J. A. Vírseda, \& J. M. Bezanilla. (Eds.). Enfermedad y Familia. México: Universidad Autónoma del Estado de México.

Devarajooh, C., \& Chinna, K. (2017). Depression, distress, and self-efficacy: the impact on diabetes self-care practices. Plos ONE, 12(3), 1-16. DOI: https://doi.org/10.1371/journal.pone.0175096

Di Clemente, C. C., Carbonari, J.P., Montgomery, R.P.G., \& Hughes, S.O. (1994). The alcohol abstinence self-efficacy scale. Journal of Studies on Alcohol, 55(2), 141-148. DOI: http://dx.doi.org/10.15288/jsa.1994.55.141

DiMatteo M. (2004). Variations in patients' adherence to medical recommendations: A quantitative review of 50 years of research. Medical Care, 42, 200-209. DOI: 10.1097/01.mlr.0000114908.90348.f9

Essery, R., Geraghty, A. W., Kirby, S., \& Yardley, L. (2017). Predictors of adherence to homebased physical therapies: a systematic review. Disability and Rehabilitation, 39(6), 519534. DOI: $10.3109 / 09638288.2016 .1153160$

Ferrer, R., Klein, W.M., Persoskie, A., Avishai-Yitshak, A., \& Sheeran, P. (2016). The tripartite model of risk perception (TRIRISK): Distinguishing deliberative, affective, and experiential components of perceived risk. The Society of Behavioral Medicine, 50, 653-

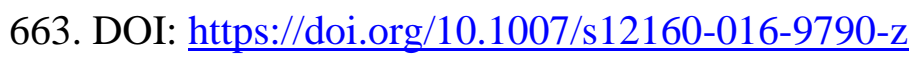

González, J. S., Shreck, E., Psaros, C., \& Safren, S. A. (2015). Distress and type 2 diabetestreatment adherence: A mediating role for perceived control. Health psychology, 34(5), 505-513. DOI: $10.1037 /$ hea0000131

Flórez, L. (2007). Psicología social de la salud promoción y prevención. Colombia: Manual Moderno. 
Relación entre autoeficacia, apoyo social, adherencia al tratamiento y HbA1C por nivel de percepción de amenaza en pacientes con DM2

González-Cantero, J, González, Ma., Vázquez, J., \& Galán, S. (2015). Autoeficacia y adherencia terapéutica en personas con diabetes mellitus tipo 2. Revista de investigación en psicología, 18(1), 47-61. DOI: https://doi.org/10.15381/rinvp.v18i1.11772

Huber, C. A., \& Reich, O. (2016). Medication adherence in patients with diabetes mellitus: does physician drug dispensing enhance quality of care? Evidence from a large health claims database in Switzerland. Patient preference and adherence, 10, 1803-1809. DOI: 10.2147/PPA.S115425

Instituto Nacional de Salud Pública. (2018). Encuesta nacional de salud y nutrición (ENSANUT) 2018, presentación de resultados. Recuperado https://ensanut.insp.mx/encuestas/ensanut2018/doctos/informes/ensanut_201 8_presentacion_resultados.pdf

Karimy, M., Araban, M., Zareban, I., Taher, M., \& Abedi, A. (2016). Determinants of adherence to self-care behavior among women with type 2 diabetes: an explanation based on health belief model. Medical Journal of The Islamic Republic of Iran, 30(386), 3-8.

King, D.K., Glasgow, R.E., Toobert, D.J., Strycker, L.A., Estabrooks, P.A., Osuna, D., \& Faber, A.J. (2010). Self-efficacy, problem solving, and social-environmental support are associated with diabetes self-management behaviors. Diabetes Care, 4, 751-753. DOI: https://doi.org/10.2337/dc09-1746

Li Cheng, M., Janet, W.H., Doris, Y.P., \& Xiaomeri, L. (2016). The association between selfmanagement barriers and self-efficacy in chinese patients with type 2 Diabetes: The mediating role appraisal. World views on evidence-based nursing, 13(5), 356-362. DOI: 10.1111/wvn. 12159

Madero, A. (2009). Análisis de validación exploratoria de la Escala de Creencias en SaludDM2/Versión pacientes geriátricos. (Tesis de Licenciatura). Recuperado de http://www.bibliotecadigital.uson.mx/pagindice.aspx?tesis=19912

Martos-Méndez, M.J. (2015). Self-efficacy and adherence to treatment: the mediating effects of social support. Journal of Behavior, Health \& Social Issues, 7(2), 19-29. DOI: http://dx.doi.org/10.5460/jbhsi.v7.2.52889

Mills, B., Reyna, V.F., \& Estrada, S. (2008). Explaining contradictory relations between risk perception and risk taking. Psychological Science in the Public Interest, 19(5), 429-433. DOI: https://doi.org/10.1111/j.1467-9280.2008.02104.x 
Nasmith, L., Cote, B., \& Cox, J. (2004). The challenge of promoting integration: conceptualization, implementation, and assessment of a pilot care delivery model for patients with type 2 diabetes. Family Medicine, 36, 40-50.

Nicklett, E.J., \& Liang, J. (2010). Diabetes-related support, regimen adherence, and health decline among older adults. The Journals of Gerontology Series B: Psychological Sciences and Social Sciences, 65(3), 390-399. DOI: https://doi.org/10.1093/geronb/gbp050

Noriega, A., García, P., Sánchez, F.M., \& Zegbe, J. (2014). Determinantes psicosociales de la adherencia al tratamiento en pacientes diabéticos tipo 2. Revista electrónica semestral en ciencias de la salud, 1, 1-19.

Ortiz, G.R. (2013). La adherencia al tratamiento. Capacidad predictiva de dos modelos sociocognitivos de creencias en salud. En G.M. Ortiz (Ed.), Tópicos Selectos en Psicología de la Salud. Aportes Latinoamericanos (51-104). México: Asociación Latinoamericana de Psicología de la Salud.

Patino, A., M, Sánchez, J., Eidson, M., \& Delamater, A.M. (2005). Health beliefs and regimen adherence in minority adolescents with type 1 diabetes. Journal of Pediatric Psychol, 30(6), 503-512. DOI: https://doi.org/10.1093/jpepsy/jsi075

Pérez, E. y Bautista, S. (2008). Susceptibilidad percibida de Diabetes Mellitus en adultos no diagnostica-dos. Revista PsicologiaCientifica.com, 10(3). Recuperado de: http://www.psicologiacientifica.com/diabetes-mellitus-susceptibilidad-percibida

Pourghaznein, T., Ghaffari, F., Hasanzadeh, F., \& Chamanzari, H. (2013). The relationship between health beliefs and medication adherence in patients with type 2 diabetes: A correlation-cross sectional study. Life Sciencie Journal, 10(4), 38-46. DOI: https://doi.org/10.1186/s40780-019-0132-8

Rouyard, T., Kent, S., Baskerville, R., Leal, J., \& Gray, A. (2017). Systematic review or metaanalysis perceptions of risks for diabetes-related complications in type 2 diabetes populations: a systematic review. Diabetic Medicine, 34, 467-477. DOI: https://doi.org/10.1111/dme.13285

Sánchez-Arellano, A.A., Navarro, G., \& Padros, F. (2017). Características psicométricas de las subescalas de susceptibilidad y severidad percibida de la ECS-DM2/PG. Revista Mexicana de Psicología. Número especial: Memorias en extenso XXV Congreso Mexicano de Psicología. Pág. 1463-1466. Puerto Vallarta, Jalisco. Octubre, 2017. ISSN 2007-9222. 
Relación entre autoeficacia, apoyo social, adherencia al tratamiento y HbA1C por nivel de percepción de amenaza en pacientes con DM2

Shreck, E., Gonzalez, J.S., Cohen, H.W., \& Walker, E.A. (2013). Risk perception and selfmanagement in urban adults with type 2 diabetes: the improving diabetes outcomes study. Journal of Behavioral Medicine, 21, 88-98. DOI: https://doi.org/10.1007/s12529-013$\underline{9291-4}$

Steed, L., Cooke, D., \& Newman, S. (2003). A systematic review of psychosocial outcomes following education, self-management, and psychological interventions in diabetes mellitus. Patient Educator Counseling, 51, 5-15. DOI: https://doi.org/10.1016/S0738$\underline{\text { 3991(02)00213-6 }}$

Strom, J.L., \& Egede, L.E. (2012). The impact of social support on outcomes in adult patients with type 2 Diabetes: A Systematic Review. Journals Current Diabetes Reports, 12, 769-781. DOI: https://doi.org/10.1007/s11892-012-0317-0

Tang, T.S., Brown, M.B., Funnell, M.M., \& Anderson, R.M. (2008). Social support, quality of life, and self-care behaviors among African Americans with type 2 diabetes. The diabetes educator, 34(2), 266-276. DOI: https://doi.org/10.1177/0145721708315680

Villalobos P.A., \& Araya C. (2001). Variables que afectan la adherencia al tratamiento en la Diabetes Mellitus tipo II y características poblacionales de una muestra de la zona norte de Costa Rica. Revista Costarricense de Psicología, 33, 19-37.

Villalobos-Pérez, A., Brenes-Sáenz, J.C., Quirós-Morales, D., \& León-Sanabria, G. (2006). Características psicométricas de la escala de adherencia al tratamiento de la diabetes mellitus tipo II-versión II (Eatdm-III) en una muestra de pacientes diabéticos de Costa Rica. Acta Colombiana de Psicología, 9(2), 31-38.

Vinaccia, S., Contreras, F., Restrepo, L.M., Cadena, F., \& Anaya, J.M. (2005). Autoeficacia, desesperanza aprendida e incapacidad funcional en pacientes con diagnóstico de artritis reumatoide. International Journal of Clinical and Health Psychology, 5(1), 129-142.

Yeong, M. (2004). The relationship of health beliefs and complication prevention behaviors of Chinese individuals with Type 2 Diabetes Mellitus. Diabetes Research and Clinical Practice, 66, 71-77. DOI: 10.1016/j.diabres.2004.02.021 